doi:10.1002/ejhf.1563

Online publish-ahead-of-print 24 July 2019

\section{Real-world versus trial patients with transthyretin amyloid cardiomyopathy}

Transthyretin (TTR) amyloid cardiomyopathy (ATTR-AC) is caused either by single-point mutations in the TTR gene (ATTRv-AC) or by deposition of the wild-type protein (ATTRwtAC). ${ }^{1}$ Long been considered a rare disease, ATTR-AC has been increasingly recognized in recent years, particularly among the elderly, ${ }^{1}$ mostly due to the possibility of a non-invasive diagnosis through bone scintigraphy. ${ }^{2}$

Recent trials have demonstrated that treatment with medications that silence TTR production or stabilize the TTR tetramer may favourably alter the progression of ATTR-AC. ${ }^{3,4}$ In particular, tafamidis, a compound that inhibits TTR dissociation, has demonstrated to improve the prognosis of both ATTRv-AC and ATTRwt-AC patients enrolled in the Transthyretin Amyloidosis Cardiomyopathy Clinical Trial (ATTR-ACT). ${ }^{3}$ However, whether the sample enrolled in the ATTR-ACT could be representative of a real-world (RW) ATTR-AC population remains unknown. To get some insight into this question, we compared the main characteristics of subjects treated with tafamidis $(n=264)$ in ATTR-ACT, ${ }^{3}$ with those of a large Italian cohort of ATTR-AC patients.

We included in the present analysis all consecutive ATTR-AC patients diagnosed according to international recommendations ${ }^{2}$ at five Italian referral centres between January 2010 and August 2018, regardless of their clinical status at diagnosis and vital status at follow-up. We excluded patients with other form of amyloid cardiomyopathy, as well as those ATTR-AC subjects enrolled in the ATTR-ACT trial (29 patients enrolled in Bologna, Florence and Pavia). ATTRv-AC patients with a prevalent neurological phenotype were also excluded according to the ATTR-ACT protocol. ${ }^{5}$ Participating centres were requested to include main patients' characteristics at first clinical evaluation in a centralized anonymized database. All patients had signed an informed consent for processing of personal data for scientific research purpose. Characteristics of the two samples were compared using one-sample $t$-test or Wilcoxon test, or test for proportion as appropriate.

Our RW sample included 507 Caucasian patients (Table 1). The distribution of ATTRv$A C$ and ATTRwt-AC was comparable in ATTR-ACT vs. RW patients, although 19 RW ones refused to undergo genetic testing. Age was similar in the two groups, but RW patients were less frequently males, and had higher prevalence of hypertension and diabetes. In addition, their clinical conditions were significantly better, including higher blood pressure and heart rate and a lower New York Heart Association (NYHA) functional class. Results of blood tests were available for more than $50 \%$ of the RW sample, and showed lower natriuretic peptide and troponin levels, and higher creatinine clearance. At echocardiography, RW patients displayed similar wall thickness, but larger left atrial dimension and a greater left ventricular ejection fraction than ATTR-ACT subjects. They were also more likely to receive cardiovascular medications, including diuretics, beta-blockers and angiotensin-converting enzyme inhibitors/angiotensin receptor blockers.

In this RW sample enrolled over the past 8 years, ATTR-AC patients at diagnosis had an apparently milder disease in terms of NYHA functional class, haemodynamics and biomarkers when compared to trial subjects. Such a healthier RW clinical profile may be the result of the greater awareness that ATTR-AC has gained in the recent years ${ }^{6}$ and of the widespread use of cardiac imaging techniques allowing an earlier and non-invasive 
Table 1 Characteristics of transthyretin amyloid cardiomyopathy trial patients at enrolment versus real-world patients at diagnosis

\begin{tabular}{|c|c|c|c|}
\hline & ATTR-ACT trial & Real-world & $P$-value \\
\hline Total population & 264 (tafamidis pooled) & 507 (Italian cohort) & \\
\hline Age (years) & $74.5 \pm 7.2$ & $74.5 \pm 8.7$ & 0.988 \\
\hline Male sex & $241(91.3)$ & $429(84.6)$ & $<0.0001$ \\
\hline Caucasian & $211(79.9)$ & $507(100)$ & $<0.0001$ \\
\hline \multicolumn{4}{|l|}{ Co-morbidities } \\
\hline Hypertension & $145(54.9)$ & $301(59.4)$ & 0.021 \\
\hline Diabetes & $20(7.6)$ & $67(13.2)$ & $<0.0001$ \\
\hline \multicolumn{4}{|l|}{ TTR genotype } \\
\hline ATTRv & $63(23.9)$ & $120(23.7)$ & 0.458 \\
\hline ATTRwt & $201(76.6)$ & $368(72.6)$ & 0.070 \\
\hline Genetics not performed & $0(0)$ & $19(3.7)$ & \\
\hline \multicolumn{4}{|l|}{ Blood pressure (supine) (mmHg) } \\
\hline SPB & $115.4 \pm 15.4$ & $130.3 \pm 18.7$ & $<0.0001$ \\
\hline DPB & $70.4 \pm 10.3$ & $76.9 \pm 10.1$ & $<0.0001$ \\
\hline Heart rate (supine) (b.p.m.) & $70.7 \pm 12.3$ & $73.1 \pm 12.7$ & $<0.0001$ \\
\hline \multicolumn{4}{|l|}{ NYHA class } \\
\hline 1 & $24(9.1)$ & $106(20.9)$ & $<0.0001$ \\
\hline II & $162(61.4)$ & $287(56.6)$ & 0.026 \\
\hline III & $78(29.5)$ & $112(22.1)$ & $<0.001$ \\
\hline IV & $0(0)$ & $2(0.4)$ & \\
\hline NT-proBNP $(\mathrm{pg} / \mathrm{mL})$ & $2995.9[1751.5-4861.5]$ & $2547[1333-4578](n=398)^{\mathrm{a}}$ & $<0.001$ \\
\hline BNP $(\mathrm{pg} / \mathrm{mL})$ [if NT-proBNP not available] & & $285[150-629](n=71)^{\mathrm{a}}$ & \\
\hline Creatinine clearance $(\mathrm{mL} / \mathrm{min})$ & $58.8 \pm 17.9$ & $66.5 \pm 25.5(n=475)^{\mathrm{a}}$ & $<0.0001$ \\
\hline Troponin I (ng/mL) & $0.14[0.09-0.20]$ & $0.02[0.01-0.07](n=294)^{\mathrm{a}}$ & $<0.0001$ \\
\hline Troponin T (ng/L) [if troponin I not available] & & $54.5(42.0-88.0)(n=78)^{\mathrm{a}}$ & \\
\hline \multicolumn{4}{|l|}{ Echocardiography } \\
\hline LA diameter (mm) & $43.8 \pm 7$ & $46.6 \pm 6.5$ & $<0.0001$ \\
\hline IVS thickness (mm) & $16.7 \pm 3.8$ & $16.9 \pm 3.0$ & 0.134 \\
\hline $\operatorname{LVEF}(\%)$ & $48.4 \pm 10.3$ & $53.4 \pm 10.7$ & $<0.0001$ \\
\hline \multicolumn{4}{|l|}{ Medications } \\
\hline Diuretics & $175(66.3)$ & $391(77.1)$ & $<0.0001$ \\
\hline Beta-blockers & $76(28.8)$ & $233(46.0)$ & $<0.0001$ \\
\hline ACEi/ARBs & $69(26.1)$ & $251(49.5)$ & $<0.0001$ \\
\hline Antithrombotics & $105(39.8)$ & 199 (39.3) & 0.818 \\
\hline
\end{tabular}

Values are given as mean \pm standard deviation, number (\%), or median [Q1-Q3].

ACEi, angiotensin-converting enzyme inhibitor; ARB, angiotensin receptor blocker; ATTR-ACT, Transthyretin Amyloidosis Cardiomyopathy Clinical Trial; ATTRv-AC, hereditary variant transthyretin amyloid cardiomyopathy; ATTRwt-AC, wild-type transthyretin amyloid cardiomyopathy; BNP, B-type natriuretic peptide; DBP, diastolic blood pressure; IVS, interventricular septum; LA, left atrial; LVEF, left ventricular ejection fraction; NT-proBNP, N-terminal pro-B-type natriuretic peptide; NYHA, New York Heart Association; SBP, systolic blood pressure; TTR, transthyretin.

a Number of patients with available data.

diagnosis of the disease. ${ }^{2}$ This could also explain the higher proportion of NYHA class I-II patients in our cohort, as already shown in other series. ${ }^{7}$ Notably, these characteristics resemble those of ATTR-ACT patients that seemed to benefit the most from the experimental treatment with tafamidis. ${ }^{3}$ On the other hand, an ATTR-AC diagnosis may increasingly be reached in individuals already 'on-treatment' for other cardiovascular conditions, such as hypertension and diabetes, which were more prevalent and probably led to a wider usage of cardiac medications in our RW population.

Tafamidis only inhibits further TTR amyloid deposition, thus it is expected to have its greater impact when administered early in the disease course. ${ }^{3}$ Accordingly, its benefits on hospitalization and survival were not immediate, but seen after approximately 12 and 18 months of treatment, respectively. ${ }^{3}$ Therefore, the possibility, as highlighted by the present report, of diagnosing ATTR$A C$ in patients mostly with few symptoms may represent a striking opportunity in order to offer tafamidis treatment to a greater number of subjects expected to benefit from it, altering significantly the course of their disease. Medications that silence TTR production have also shown some preliminary positive results, but their investigation so far has been limited to ATTRv-AC patients of younger age and with milder disease as compared to the ATTR-ACT and RW ones analysed here. ${ }^{4}$ Interestingly, the pre-defined proportion of ATTRv/ATTRwt-AC of ATTR-ACT was replicated by chance in our RW population, 
in which ATTRv-AC was mostly related to Ile68Leu, Val122lle and Val30Met late-onset mutations.

In the context of cardiovascular medicine, it is very unusual to see a RW population being clinically comparable to (or even more fit than) a trial population; thus, we did not anticipate these results. However, some limitations should be acknowledged. A shorter duration of the ATTR-AC disease could explain the RW milder phenotype; however, this information was neither available from ATTR-ACT nor from our database. In addition, our RW sample may have suffered from substantial referral bias, as ATTR-AC patients with a greater burden of co-morbidities and a lower life expectancy might less likely be referred to dedicated centres for more advanced diagnostics and treatments. Nonetheless, the RW population included in this analysis might represent a sizable indicator of patients suitable for a dedicated specific treatment in our country. Finally, we did not systematically collect important parameters that were considered as exclusion criteria in ATTR-ACT, namely a low modified body mass index and a short 6-min walk test distance. ${ }^{3}$ These indicators of poor prognosis should always be assessed before considering a patient for expensive therapies with survival benefits expected only at long-term follow-up.

In conclusion, our findings that RW ATTRAC patients closely resemble those enrolled in ATTR-ACT have relevant implications, considering that ATTR-AC is still considered a rare condition, and specific treatments will likely become initially available for a small proportion of the affected patients, primarily because of the relevant costs.
Conflict of interest: M.C. served on the Italian scientific advisory board of Pfizer. F.C. served on the Italian scientific advisory board of AKCEA, and received an unrestricted research grant from Pfizer. A.M. received personal fees from Alnylam Pharmaceuticals. C.A. served on the Italian scientific advisory board of Pfizer. F.P. served on the Italian scientific advisory board of Alnylam Pharmaceuticals and Pfizer. C.R. served on the Italian scientific advisory board of Pfizer, received unrestricted research grants and personal fees from Pfizer, and personal fees from Alnylam Pharmaceuticals. S.P. served on the Italian scientific advisory board of Pfizer. The other authors report no conflicts of interest.

\section{Marco Canepa ${ }^{1,2, *}$, Giacomo Tini ${ }^{1,2}$, Beatrice Musumeci ${ }^{3}$, \\ Francesco Cappelli ${ }^{4}$, Agnese Milandri ${ }^{5}$, Roberta Mussinelli ${ }^{6}$, Camillo Autore ${ }^{3}$, Federico Perfetto ${ }^{4}$, Claudio Rapezzi ${ }^{5}$, and Stefano Perlini ${ }^{6}$}

${ }^{1}$ Cardiovascular Disease Unit, IRCCS Ospedale Policlinico San Martino - IRCCS Italian Cardiovascular Network, Genova, Italy; ${ }^{2}$ Department of Internal Medicine, University of Genova, Genova, Italy; ${ }^{3}$ Cardiology Unit, Clinical and Molecular Medicine Department, Faculty of Medicine and Psychology, Sapienza University of Rome, Rome, Italy; ${ }^{4}$ Tuscan Regional Amyloid Center, Careggi University Hospital, Florence, Italy; ${ }^{5}$ Cardiology, Department of Experimental, Diagnostic and Specialty Medicine, Alma Mater University of Bologna, Bologna, Italy; and ${ }^{6}$ Emergency Medicine, Department of Internal Medicine, Amyloidosis Research and Treatment Center, Fondazione IRCCS Policlinico San Matteo, University of Pavia, Pavia, Italy

Email: marco.canepa@unige.it

\section{References}

1. Maurer MS, Elliott P, Comenzo R, Semigran M, Rapezzi $C$. Addressing common questions encountered in the diagnosis and management of cardiac amyloidosis. Circulation 2017;135:1357-1377.

2. Gillmore JD, Maurer MS, Falk RH, Merlini G, Damy T, Dispenzieri A, Wechalekar AD, Berk JL, Quarta CC, Grogan M, Lachmann HJ, Bokhari S, Castano A, Dorbala S, Johnson GB, Glaudemans AW, Rezk T, Fontana M, Palladini G, Milani P, Guidalotti PL, Flatman K, Lane T, Vonberg FW, Whelan CJ, Moon JC, Ruberg FL, Miller EJ, Hutt DF, Hazenberg BP, Rapezzi C, Hawkins PN. Nonbiopsy diagnosis of cardiac transthyretin amyloidosis. Circulation 2016;133: 2404-2412.

3. Maurer MS, Schwartz JH, Gundapaneni B, Elliott PM, Merlini G, Waddington-Cruz M, Kristen AV, Grogan M, Witteles R, Damy T, Drachman BM, Shah SJ, Hanna M, Judge DP, Barsdorf Al, Huber P, Patterson TA, Riley S, Schumacher J, Stewart M, Sultan MB, Rapezzi C; ATTR-ACT Study Investigators. Tafamidis treatment for patients with transthyretin amyloid cardiomyopathy. N Engl J Med 2018;379:1007-1016.

4. Solomon SD, Adams D, Kristen A, Grogan M, Gonzalez-Duarte A, Maurer MS, Merlini G, Damy T, Slama MS, Brannagan TH 3rd, Dispenzieri A, Berk JL, Shah AM, Garg P, Vaishnaw A, Karsten V, Chen J, Gollob J, Vest J, Suhr O. Effects of patisiran, an RNA interference therapeutic, on cardiac parameters in patients with hereditary transthyretin-mediated amyloidosis. Circulation 2019;139:431-443.

5. Maurer MS, Elliott P, Merlini G, Shah SJ, Cruz MW, Flynn A, Gundapaneni B, Hahn C, Riley S, Schwartz J, Sultan MB, Rapezzi C; ATTR-ACT Study Investigators. Design and rationale of the phase 3 ATTR-ACT clinical trial (Tafamidis in Transthyretin Cardiomyopathy Clinical Trial). Circ Heart Fail 2017;10:e03815.

6. Maurer MS, Sultan MB, Rapezzi C. Tafamidis for transthyretin amyloid cardiomyopathy. N Engl J Med 2019;380:196-197.

7. Gonzalez-Lopez E, Gagliardi C, Dominguez F, Quarta CC, de Haro-Del Moral FJ, Milandri A, Salas C, Cinelli M, Cobo-Marcos M, Lorenzini M, Lara-Pezzi E, Foffi S, Alonso-Pulpon L, Rapezzi C, Garcia-Pavia P. Clinical characteristics of wild-type transthyretin cardiac amyloidosis: disproving myths. Eur Heart J 2017;38:1895-1904. 